\title{
TOPOLOGICAL FULL GROUPS OF MINIMAL SUBSHIFTS AND JUST-INFINITE GROUPS
}

\author{
Dedicated to the memory of Greg Hjorth \\ SIMON THOMAS \\ Mathematics Department, Rutgers University \\ New Brunswick, New Jersey 08854, USA \\ E-mail: sthomas@math.rutgers.edu
}

\begin{abstract}
Using recent work on the algebraic structure of topological full groups of minimal subshifts, we prove that the isomorphism relation on the space of infinite finitely generated simple amenable groups is not smooth. As an application, we deduce that there does not exist an isomorphism-invariant Borel map which selects a just-infinite quotient of each infinite finitely generated group.
\end{abstract}

Keywords: Borel equivalence relation; Topological full group; Minimal subshift; Just-infinite group.

\section{Introduction}

In [24], confirming a conjecture of Hjorth-Kechris [16], Thomas-Velickovic proved that the isomorphism relation on the space $\mathcal{G}_{f g}$ of finitely generated groups is a universal countable Borel equivalence relation. (Here $\mathcal{G}_{f g}$ denotes the Polish space of finitely generated groups introduced by Grigorchuk [12]; i.e. the elements of $\mathcal{G}_{f g}$ are the isomorphism types of marked groups $(G, \bar{c})$, where $G$ is a finitely generated group and $\bar{c}$ is a finite sequence of generators.) This result suggests the project of analyzing the Borel complexity of the isomorphism relation for various restricted classes of finitely generated groups; and the main result in this paper can be regarded as the first step in this analysis for both the class of infinite finitely generated simple groups and the class of infinite finitely generated amenable groups.

Theorem 1.1. The isomorphism relation on the space of infinite finitely generated simple amenable groups is not smooth.

The proof of Theorem 1.1 makes use of some recent work of GiordanoPutnam-Skau [11], Bezuglyi-Medynets [1], Matui [20] and Juschenko- 
Monod [18] on the topological full groups of minimal subshifts. More precisely, if $X \subseteq n^{\mathbb{Z}}$ is a minimal subshift and $T F(X)$ is the topological full group, then the commutator subgroup $T F(X)^{\prime}$ is an infinite finitely generated simple amenable group. Furthermore, if $Y \subseteq n^{\mathbb{Z}}$ is another minimal subshift, then $T F(X)^{\prime} \cong T F(Y)^{\prime}$ if and only if $X$ and $Y$ are flip conjugate. (A fuller discussion, including the relevant definitions, will be presented in Section 3.) Hence, in order to prove Theorem 1.1, it is enough to show that the flip conjugacy relation for minimal subshifts $X \subseteq n^{\mathbb{Z}}$ is not smooth. In Section 4, we will prove the stronger result that the flip conjugacy relation for Toeplitz subshifts is not smooth. In [3], Clemens showed that the topological conjugacy relation for arbitrary subshifts $X \subseteq n^{\mathbb{Z}}$ is a universal countable Borel equivalence relation. Unfortunately the subshifts constructed by Clemens are very far from minimal; and it is currently not known whether or not the topological conjugacy relation for minimal subshifts is strictly more complex than the Vitali equivalence relation $E_{0}$. However, it still seems reasonable to conjecture that the following strengthening of Theorem 1.1 should be true.

Conjecture 1.2. The isomorphism relation on the space of infinite finitely generated simple amenable groups is countable universal.

It should be pointed out that it is currently not known whether or not the isomorphism relation on the space $\mathcal{G}_{a m}$ of infinite finitely generated amenable groups or on the space $\mathcal{G}_{\text {sim }}$ of infinite finitely generated simple groups is countable universal. Of course, it is also natural to consider the complexity of the isomorphism relation on the space $\mathcal{G}_{k a z}$ of finitely generated Kazhdan groups.

Conjecture 1.3. The isomorphism relation on $\mathcal{G}_{k a z}$ is not smooth.

Here it is worthwhile pointing out that a result of Ol'shanskii [21] implies that if $G$ is any countable group, then there exists a finitely generated Kazhdan group $K$ such that $G$ embeds into $K$. More precisely, let $H$ be an infinite hyperbolic Kazhdan group. (Such a group is necessarily finitely presented and hence finitely generated. For example, see Bridson-Haefliger [2, Proposition III.Г.2.2].) Then, by Ol'shanskii [21], if $G$ is any countable group, then $G$ embeds into a quotient $H / N$ of $H$; and, since the class of Kazhdan groups is closed under the taking of quotients, it follows that $H / N$ is a finitely generated Kazhdan group. This suggests that the isomorphism relation on $\mathcal{G}_{k a z}$ is also a universal countable Borel equivalence relation. 
In Section 5, we will present an application of Theorem 1.1 to the theory of just-infinite groups. Here an infinite group $\Gamma$ is said to be just-infinite if every nontrivial normal subgroup of $\Gamma$ has finite index. In [13], Grigorchuk observed that if $G$ is an infinite finitely generated group, then $G$ has a just-infinite homomorphic image. To see this, consider the poset

$$
\mathbb{P}=\{N \unlhd G \mid[G: N]=\infty\},
$$

partially ordered by inclusion. If $\left\{N_{i} \mid i \in I\right\}$ is a chain in $\mathbb{P}$, then $N=\bigcup_{i \in I} N_{i} \in \mathbb{P}$, since otherwise $[G: N]<\infty$ and so $N$ is finitely generated, which is a contradiction. Hence, by Zorn's Lemma, there exists a maximal element $N \in \mathbb{P}$ and clearly $G / N$ is just-infinite. Of course, if $G$ is an explicitly given infinite finitely generated group, then it is not necessary to use Zorn's Lemma in order to construct a just-infinite homomorphic image. More precisely, as we will explain in Section 2, there exists a Borel map $\theta: \mathcal{G}_{f g} \rightarrow \mathcal{G}_{f g}$ such that if $(G, \bar{c}) \in \mathcal{G}_{f g}$ is infinite, then $\theta(G, \bar{c})$ is a just-infinite homomorphic image of $G$. However, as our notation suggests, the definition of the just-infinite group $\theta(G, \bar{c})$ depends essentially upon the finite sequence of generators $\bar{c}$ and it is natural to ask whether there exists such a Borel map $\theta$ with the property that the isomorphism type of the just-infinite group $\varphi(G, \bar{c})$ only depends upon the isomorphism type of $G$. In Section 5, we will use Theorem 1.1 to show that no such map exists.

Theorem 1.4. There does not exist a Borel map $\theta: \mathcal{G}_{f g} \rightarrow \mathcal{G}_{f g}$ such that for all infinite $(G, \bar{c}),(H, \bar{d}) \in \mathcal{G}_{f g}$,

(i) $\theta(G, \bar{c})$ is a just-infinite homomorphic image of $G$; and

(ii) if $G \cong H$, then $\theta(G, \bar{c}) \cong \theta(H, \bar{d})$.

The remainder of this paper is organized as follows. In Section 2, we will recall some basic notions and results from the theory of countable Borel equivalence relations, including the definition of the space $\mathcal{G}_{f g}$ of (marked) finitely generated groups. In Section 3, we will discuss some recent results concerning the structure of topological full groups of minimal subshifts; and in Section 4, we will prove that the flip conjugacy relation for Toeplitz subshifts is not smooth and hence also that the isomorphism relation on the space of infinite finitely generated simple amenable groups is not smooth. Finally, in Section 5, we will present the proof of Theorem 1.4.

\section{Countable Borel equivalence relations}

In this section, we will recall some basic notions and results from the theory of countable Borel equivalence relations, including the definition of the 
space $\mathcal{G}_{f g}$ of (marked) finitely generated groups.

Suppose that $(X, \mathcal{B})$ is a measurable space; i.e. that $\mathcal{B}$ is a $\sigma$-algebra of subsets of the set $X$. Then $(X, \mathcal{B})$ is said to be a standard Borel space if there exists a Polish topology $\mathcal{T}$ on $X$ such that $\mathcal{B}$ is the $\sigma$-algebra of Borel subsets of $(X, \mathcal{T})$. If $X, Y$ are standard Borel spaces, then a map $f: X \rightarrow Y$ is Borel if $f^{-1}(Z)$ is a Borel subset of $X$ for each Borel subset $Z \subseteq Y$. Equivalently, $f: X \rightarrow Y$ is Borel if $\operatorname{graph}(f)$ is a Borel subset of $X \times Y$.

Let $X$ be a standard Borel space. Then a Borel equivalence relation on $X$ is an equivalence relation $E \subseteq X^{2}$ which is a Borel subset of $X^{2}$. If $E, F$ are Borel equivalence relations on the standard Borel spaces $X, Y$ respectively, then a Borel map $f: X \rightarrow Y$ is said to be a homomorphism from $E$ to $F$ if for all $x, y \in X$,

$$
x E y \quad \Longrightarrow \quad f(x) F f(y) \text {. }
$$

If $f$ satisfies the stronger property that for all $x, y \in X$,

$$
x E y \quad \Longleftrightarrow \quad f(x) F f(y),
$$

then $f$ is said to be a Borel reduction and we write $E \leq_{B} F$. If both $E \leq_{B} F$ and $F \leq_{B} E$, then $E$ and $F$ are said to be Borel bireducible and we write $E \sim_{B} F$. Finally we write $E<_{B} F$ if both $E \leq_{B} F$ and $F \not{ }_{B} E$.

In this paper, we will only be concerned with countable Borel equivalence relations; i.e. Borel equivalence relations $E$ such that every $E$-equivalence class is countable. A detailed development of the general theory of countable Borel equivalence relations can be found in Jackson-Kechris-Louveau [17]. Here we will only recall some of the most basic results of the theory.

With respect to Borel reducibility, the least complex countable Borel equivalence relations are those which are smooth; i.e. those countable Borel equivalence relations $E$ on a standard Borel space $X$ such that $E$ is Borel reducible to the identity relation $\operatorname{Id}_{Y}$ on some (equivalently every) uncountable standard Borel space $Y$. Next in complexity come those countable Borel equivalence relations $E$ which are Borel bireducible with the Vitali equivalence relation $E_{0}$, which is defined on the space $2^{\mathbb{N}}$ of infinite binary sequences by

$$
x E_{0} y \quad \Longleftrightarrow \quad x(n)=y(n) \text { for all but finitely many } n .
$$

More precisely, by Harrington-Kechris-Louveau [15], if $E$ is any (not necessarily countable) Borel equivalence relation, then $E$ is nonsmooth if and only if $E_{0} \leq_{B} E$. It turns out that there is also a most complex countable Borel equivalence relation $E_{\infty}$, which is universal in the sense that 
$F \leq_{B} E_{\infty}$ for every countable Borel equivalence relation $F$. (Clearly this universality property uniquely determines $E_{\infty}$ up to Borel bireducibility.) Furthermore, $E_{\infty}$ is strictly more complex than $E_{0}$. The universal countable Borel relation $E_{\infty}$ has a number of natural realizations in many areas of mathematics, including algebra, topology and recursion theory. In particular, by Thomas-Velickovic [24], the isomorphism relation $\cong$ on the space $\mathcal{G}_{f g}$ of finitely generated groups is a universal countable Borel equivalence relation.

Most of our effort in this paper will be devoted to proving that various countable Borel equivalence relations are nonsmooth. Here we will make use of the following two observations.

Proposition 2.1. If $E \subseteq F$ are countable Borel equivalence relations on the standard Borel space $X$ and $E$ is nonsmooth, then $F$ is also nonsmooth.

Proof. This is an easy consequence of the Feldman-Moore Theorem [8]. (For example, see Thomas [22, Lemma 2.1].)

If $E, E^{\prime}$ are countable Borel equivalence relations on the standard Borel spaces $Z, Z^{\prime}$, then $E$ is said to be weakly Borel reducible to $E^{\prime}$ if there exists a countable-to-one Borel homomorphism $f: X \rightarrow X^{\prime}$ from $E$ to $E^{\prime}$. In this case, we write $E \leq_{B}^{w} E^{\prime}$.

Proposition 2.2. If $E$ is a countable Borel equivalence relation on the standard Borel space $X$ and $E_{0} \leq_{B}^{w} E$, then $E$ is nonsmooth.

Proof. By Thomas [23, Theorem 4.4], if $E_{0} \leq_{B}^{w} E$, then there exists a countable Borel equivalence relation $E^{\prime} \subseteq E$ such that $E_{0} \leq_{B} E^{\prime}$. Hence the result follows from Proposition 2.1.

In the remainder of this section, we will present a brief discussion of the Polish space $\mathcal{G}_{f g}$ of (marked) finitely generated groups, which is defined as follows. A marked group $(G, \bar{s})$ consists of a finitely generated group with a distinguished sequence $\bar{s}=\left(s_{1}, \cdots, s_{m}\right)$ of generators. (Here the sequence $\bar{s}$ is allowed to contain repetitions and we also allow the possibility that the sequence contains the identity element.) Two marked groups $\left(G,\left(s_{1}, \cdots, s_{m}\right)\right)$ and $\left(H,\left(t_{1}, \cdots, t_{n}\right)\right)$ are said to be isomorphic if $m=n$ and the map $s_{i} \mapsto t_{i}$ extends to a group isomorphism between $G$ and $H$.

Definition 2.3. For each $m \geq 2$, let $\mathcal{G}_{m}$ be the set of isomorphism types of marked groups $\left(G,\left(s_{1}, \cdots, s_{m}\right)\right)$ with $m$ distinguished generators. 
Let $\mathbb{F}_{m}$ be the free group on the generators $\left\{x_{1}, \cdots, x_{m}\right\}$. Then for each marked group $\left(G,\left(s_{1}, \cdots, s_{m}\right)\right)$, we can define an associated epimorphism $\theta_{G, \bar{s}}: \mathbb{F}_{m} \rightarrow G$ by $\theta_{G, \bar{s}}\left(x_{i}\right)=s_{i}$. It is easily checked that two marked groups $\left(G,\left(s_{1}, \cdots, s_{m}\right)\right)$ and $\left(H,\left(t_{1}, \cdots, t_{m}\right)\right)$ are isomorphic if and only if $\operatorname{ker} \theta_{G, \bar{s}}=\operatorname{ker} \theta_{H, \bar{t}}$. Thus we can naturally identify $\mathcal{G}_{m}$ with the set $\mathcal{N}_{m}$ of normal subgroups of $\mathbb{F}_{m}$. Note that $\mathcal{N}_{m}$ is a closed subset of the compact space $2^{\mathbb{F}_{m}}$ of all subsets of $\mathbb{F}_{m}$ and so $\mathcal{N}_{m}$ is also a compact space. Hence, via the above identification, we can regard $\mathcal{G}_{m}$ as a compact space.

For each $m \geq 2$, there is a natural embedding of $\mathcal{N}_{m}$ into $\mathcal{N}_{m+1}$ defined by

$$
N \mapsto \text { the normal closure of } N \cup\left\{x_{m+1}\right\} \text { in } \mathbb{F}_{m+1} \text {. }
$$

Thus we can identify $\mathcal{N}_{m}$ with the clopen subset $\left\{N \in \mathcal{N}_{m+1} \mid x_{m+1} \in N\right\}$ of $\mathcal{N}_{m+1}$ and form the locally compact Polish space $\mathcal{N}_{f g}=\bigcup \mathcal{N}_{m}$. Note that $\mathcal{N}_{f g}$ can be identified with the space of normal subgroups $N$ of the free group $\mathbb{F}_{\infty}$ on countably many generators such that $N$ contains all but finitely many elements of the basis $B=\left\{x_{i} \mid i \in \mathbb{N}^{+}\right\}$. Similarly, we can form the locally compact Polish space $\mathcal{G}_{f g}=\bigcup \mathcal{G}_{m}$ of finitely generated groups via the corresponding natural embedding

$$
\left(G,\left(s_{1}, \cdots, s_{m}\right)\right) \mapsto\left(G,\left(s_{1}, \cdots, s_{m}, 1\right)\right)
$$

From now on, we will identify $\mathcal{G}_{m}$ and $\mathcal{N}_{m}$ with the corresponding clopen subsets of $\mathcal{G}_{f g}$ and $\mathcal{N}_{f g}$. If $\Gamma \in \mathcal{G}_{f g}$, then we will write $\Gamma=\left(G,\left(s_{1}, \cdots, s_{m}\right)\right)$, where $m$ is the least integer such that $\Gamma \in \mathcal{G}_{m}$. Following the usual convention, we will completely identify the Polish spaces $\mathcal{G}_{f g}$ and $\mathcal{N}_{f g}$; and we will work with whichever space is most convenient in any given context.

For example, to see that there exists a Borel map $\theta: \mathcal{G}_{f g} \rightarrow \mathcal{G}_{f g}$ such that if $(G, \bar{c}) \in \mathcal{G}_{f g}$ is infinite, then $\theta(G, \bar{c})$ is a just-infinite homomorphic image of $G$, it is convenient to work with $\mathcal{N}_{f g}$ as follows. Suppose that $(G, \bar{c}) \in \mathcal{G}_{f g}$ is infinite and that $N \in \mathcal{N}_{f g}$ is the corresponding normal subgroup of $\mathbb{F}_{\infty}$. Then, working with a fixed enumeration $\left\{w_{k} \mid k \in \mathbb{N}\right\}$ of $\mathbb{F}_{\infty}$, we can define an increasing sequence of normal subgroups $N_{k} \in \mathcal{N}_{f g}$ by:

- $N_{0}=N$.

- $N_{k+1}$ is the normal closure $M_{k}$ of $N_{k} \cup\left\{w_{k}\right\}$ in $\mathbb{F}_{\infty}$ if $\left[\mathbb{F}_{\infty}: M_{k}\right]=\infty$. Otherwise, $N_{k+1}=N_{k}$.

Finally let $N_{\omega}=\bigcup_{k \in \mathbb{N}} N_{k}$. Then $\mathbb{F}_{\infty} / N_{\omega}$ is a just-infinite homomorphic image of $G$ and the map $N \mapsto N_{\omega}$ is Borel. 
In the remaining sections of this paper, the symbol $\cong$ will always denote the usual isomorphism relation on the space $\mathcal{G}_{f g}$ of finitely generated groups; i.e. two marked groups are $\cong$-equivalent if their underlying groups (obtained by forgetting about the distinguished sequences of generators) are isomorphic. Finally, we should mention that we will occasionally slightly abuse notation and denote the elements of $\mathcal{G}_{f g}$ by $G, H$, etc. instead of the more accurate $(G, \bar{c}),(H, \bar{d})$, etc.

\section{Topological full groups of minimal subshifts}

In this section, we will discuss some recent results concerning the structure of topological full groups of Cantor minimal systems. Let $(X, T)$ be a Cantor dynamical system; i.e. $X$ is a Cantor set and $T: X \rightarrow X$ is a homeomorphism. Then $(X, T)$ is said to be a Cantor minimal system if $X$ has no nonempty proper closed $T$-invariant subsets. It is well-known that a Cantor dynamical system $(X, T)$ is minimal if and only if $X$ is the closure of the orbit of an almost periodic point $x \in X$; and, in this case, every point $x \in X$ is almost periodic.

Definition 3.1. If $(X, T)$ is a Cantor dynamical system, then the point $x \in X$ is almost periodic if for every open neighborhood $U$ of $x$, the set

$$
R=\left\{\ell \in \mathbb{Z} \mid T^{\ell}(x) \in U\right\}
$$

of return times has bounded gaps; i.e. there exists a fixed $d \geq 1$ such that for all $z \in \mathbb{Z}$,

$$
R \cap\{z, z+1, \cdots, z+d\} \neq \emptyset
$$

Since Cantor minimal systems are infinite, it follows that they do not contain any genuinely periodic points; i.e. points with finite orbits.

Definition 3.2. If $(X, T)$ is a Cantor minimal system, then the topological full group $[[T]]$ is the group of all homeomorphisms $\pi: X \rightarrow X$ such that there exists a partition $X=C_{1} \sqcup \cdots \sqcup C_{m}$ into clopen subsets and $\ell_{1}, \cdots, \ell_{m} \in \mathbb{Z}$ such that $\pi\left\lceil C_{i}=T^{\ell_{i}}\left\lceil C_{i}\right.\right.$ for each $1 \leq i \leq m$.

The Cantor minimal systems $(X, T)$ and $(Y, S)$ are said to be topologically conjugate if there exists a homeomorphism $\pi: X \rightarrow Y$ such that $\pi \circ T=S \circ \pi$. If $(X, T)$ is topologically conjugate to either $(Y, S)$ or $\left(Y, S^{-1}\right)$, then $(X, T)$ and $(Y, S)$ are said to be flip conjugate. The following theorem combines the work of Giordano-Putnam-Skau [11] and Bezuglyi-Medynets [1]. 
Theorem 3.3. If $(X, T),(Y, S)$ are Cantor minimal systems, then the following are equivalent.

(i) $(X, T),(Y, S)$ are flip conjugate.

(ii) The topological full groups $[[T]],[[S]]$ are isomorphic as abstract groups.

(iii) The commutator subgroups $[[T]]^{\prime}$, $[[S]]^{\prime}$ are isomorphic as abstract groups.

If $n \geq 2$, then the shift transformation $\sigma$ on the Cantor space $n^{\mathbb{Z}}$ is defined by $\sigma(x)_{k}=x_{k+1}$. An infinite subset $X \subseteq n^{\mathbb{Z}}$ is said to be a subshift if $X$ is a closed $\sigma$-invariant subset. The subshift $X$ is minimal if the corresponding Cantor dynamical system $(X, \sigma)$ is minimal. In this case, we also say that $(X, \sigma)$ is a minimal subshift. The following theorem is due to Matui [20].

Theorem 3.4. Let $(X, T)$ be a Cantor minimal system.

(a) The commutator subgroup $[[T]]^{\prime}$ is an infinite simple group.

(b) The commutator subgroup $[[T]]^{\prime}$ is finitely generated if and only if $(X, T)$ is topologically conjugate to a minimal subshift over a finite alphabet.

The following result, which confirms a conjecture of GrigorchukMedynets [14], was recently proved by Juschenko-Monod [18].

Theorem 3.5. The topological full group of any Cantor minimal system is amenable.

The following result is now an immediate consequence of Theorem 3.4 and Theorem 3.5.

Corollary 3.6. If the Cantor minimal system $(X, T)$ is topologically conjugate to a minimal subshift over a finite alphabet, then the commutator subgroup $[[T]]^{\prime}$ is an infinite finitely generated simple amenable group.

Throughout this paper, the collection $\mathcal{M}_{n}$ of minimal subshifts $X \subseteq n^{\mathbb{Z}}$ will be regarded as a subspace of the standard Borel space $K\left(n^{\mathbb{Z}}\right)$ of closed subspaces of $n^{\mathbb{Z}}$. (This corresponds to identifying each minimal subshift $X$ with the corresponding pruned tree $T_{X} \subseteq \bigcup_{k \in \mathbb{N}} n^{[-k, k]}$ such that $X$ is the set $\left[T_{X}\right]$ of infinite branches through $T_{X}$.) Recall that a subshift $X \subseteq n^{\mathbb{Z}}$ is minimal if and only if some (equivalently every) point $x \in X$ is almost periodic. It follows easily that $\mathcal{M}_{n}$ is a Borel subset of $K\left(n^{\mathbb{Z}}\right)$ and hence 
that $\mathcal{M}_{n}$ is a standard Borel space. We will make use of the following result in Section 4.

Proposition 3.7. The topological conjugacy relation $E_{t c}$ and the flip conjugacy relation $E_{f p}$ are both countable Borel equivalence relations on $\mathcal{M}_{n}$.

Proof. By Clemens [3, Lemma 9], $E_{t c}$ is a countable Borel equivalence relation on $\mathcal{M}_{n}$; and, of course, this implies that $E_{f p}$ is also a countable Borel equivalence relation.

From now on, if $(X, \sigma)$ is a minimal subshift, then we will write $T F(X)$ for the corresponding topological full group.

Theorem 3.8. For each $n \geq 2$, there exists a Borel map $X \mapsto G_{X}$ from $\mathcal{M}_{n}$ to $\mathcal{G}_{f g}$ such that $G_{X} \cong T F(X)^{\prime}$.

Let $X \in \mathcal{M}_{n}$ be a minimal subshift. In order to define the (marked) group $G_{X} \in \mathcal{G}_{f g}$, we first need to explicitly describe a finite generating set for $T F(X)^{\prime}$. The following set of generators was originally extracted from Matui [20, Section 5] by Grigorchuk and Medynets in an early version of their paper [14].

Definition 3.9. Suppose that $A \subseteq X$ is a clopen subset such that the sets $A, \sigma(A)$ and $\sigma^{2}(A)$ are pairwise disjoint. Then the homeomorphism $\gamma_{A} \in T F(X)$ is defined by

$$
\gamma_{A}(x)= \begin{cases}\sigma(x) & \text { if } x \in A \cup \sigma(A) ; \\ \sigma^{-2}(x) & \text { if } x \in \sigma^{2}(A) ; \\ x & \text { otherwise. }\end{cases}
$$

By Matui [20, Section 5], each such homeomorphism $\gamma_{A}$ is an element of $T F(X)^{\prime}$; and, furthermore, $T F(X)^{\prime}$ is generated by a suitably chosen finite subset of these homeomorphisms. In more detail, for each $m \geq 1$, let $\mathcal{B}_{m}(X)$ be the set of all $m$-blocks that occur in sequences $x \in X$; i.e. the words of the form

$$
x\left\lceil[k, k+m-1]=x_{k} x_{k+1} \cdots x_{k+m-1}\right.
$$

for some $x \in X$ and $k \in \mathbb{Z}$. And for each $w \in \mathcal{B}_{m}(X)$ and $k \in \mathbb{Z}$, let

$$
S_{k}(w)=\{x \in X \mid x\lceil[k, k+m-1]=w\} .
$$


Then there exists an integer $m_{0} \geq 1$ such that for each $w \in \mathcal{B}_{m_{0}}(X), k \in \mathbb{Z}$ and $1 \leq i \leq 4$,

$$
\sigma^{i}\left(S_{k}(w)\right) \cap S_{k}(w)=S_{k-i}(w) \cap S_{k}(w)=\emptyset .
$$

(If not, then an easy compactness argument yields an element $x \in X$ such that $\sigma^{i}(x)=x$ for some $1 \leq i \leq 4$, which contradicts the fact that minimal subshifts contain no periodic points.) Finally, as pointed out by Grigorchuk and Medynets, the proof of Matui [20, Theorem 5.4] shows that the following result holds.

Proposition 3.10. If $(X, \sigma)$ is a minimal subshift, then $T F(X)^{\prime}$ is generated by $D_{m_{0}}=\left\{\gamma_{S_{0}(w)} \mid w \in \mathcal{B}_{m_{0}+3}(X)\right\}$.

We are now ready to present the proof of Theorem 3.8. Suppose that $X \in \mathcal{M}_{n}$ is a minimal subshift. Then, in a Borel manner, we can choose an integer $m_{0} \geq 1$ such that $D_{m_{0}}=\left\{\gamma_{S_{0}(w)} \mid w \in \mathcal{B}_{m_{0}+3}(X)\right\}$ generates $T(X)^{\prime}$, together with an ordering $\varphi_{1}, \cdots, \varphi_{t}$ of the elements of $D_{m_{0}}$. Also, again in a Borel manner, we can choose an element $p_{X} \in X$. (For example, see Kechris [19, Theorem 12.13].) Let $X \mapsto N_{X} \in \mathcal{N}_{t} \subseteq \mathcal{N}_{f g}$ be the Borel map defined by

$$
w\left(x_{1}, \cdots, x_{t}\right) \in N_{X} \Longleftrightarrow w\left(\varphi_{1}, \cdots, \varphi_{t}\right)\left(\sigma^{n}\left(p_{X}\right)\right)=\sigma^{n}\left(p_{X}\right) \text { for all } n \in \mathbb{Z} \text {. }
$$

Since $\left\{\sigma^{n}\left(p_{X}\right) \mid n \in \mathbb{Z}\right\}$ is dense in $X$ and each $\varphi_{i}$ is a homeomorphism, it follows that $\mathbb{F}_{t} / N_{X} \cong T F(X)^{\prime}$. This completes the proof of Theorem 3.8.

\section{Toeplitz subshifts}

Combining Theorem 3.3, Corollary 3.6 and Theorem 3.8, we see that in order to prove that the isomorphism relation on the space of infinite finitely generated simple amenable groups is not smooth, it is enough to show that the flip conjugacy relation $E_{f c}$ on the space $\mathcal{M}_{n}$ of minimal subshifts $X \subseteq n^{\mathbb{Z}}$ is not smooth. In [10, Section 9.3], Gao-Jackson-Seward proved that for each $n \geq 2$, the topological conjugacy relation $E_{t c}$ on $\mathcal{M}_{n}$ is not smooth. Applying Proposition 2.1, since $E_{t c}, E_{f c}$ are countable Borel equivalence relations and $E_{t c} \subseteq E_{f c}$, it follows that the flip conjugacy relation $E_{f c}$ on $\mathcal{M}_{n}$ is also not smooth.

Since Gao-Jackson-Seward [10] work in the more general setting of minimal free $G$-subflows, where $G$ is an arbitrary countably infinite group, their proofs are necessarily technically complex. (In fact, the existence of a single free $G$-flow for an arbitrary countably infinite group $G$ has only recently been established by Gao-Jackson-Seward [9].) In this section, we 
will present an elementary proof that the topological conjugacy relation $E_{t c}$ on the space $\mathcal{M}_{n}$ of minimal subshifts $X \subseteq n^{\mathbb{Z}}$ is not smooth.

Definition 4.1. An element $x \in n^{\mathbb{Z}}$ is said to be a Toeplitz sequence if for all $a \in \mathbb{Z}$, there exists $b \in \mathbb{N}^{+}$such that $x(a+k b)=x(a)$ for all $k \in \mathbb{Z}$.

Clearly if $x \in n^{\mathbb{Z}}$ is a Toeplitz sequence, then $x$ is almost periodic. Hence, if $x$ is a nonperiodic Toeplitz sequence, then the closure $X$ of its orbit is a minimal subshift of $n^{\mathbb{Z}}$; and, in this case, $X$ is said to be a Toeplitz flow. By Downarowicz [6, Theorem 5.1], if $X$ is a Toeplitz flow, then the set of all Toeplitz sequences $z \in X$ is a dense $G_{\delta}$ subset of $X$. It follows that the set $\mathcal{T}_{n} \subseteq \mathcal{M}_{n}$ of Toeplitz flows is a Borel subset of $\mathcal{M}_{n}$ and hence is a standard Borel space. (For example, see Kechris [19, Section 16.A].) The remainder of this section is devoted to the proof of the following result.

Theorem 4.2. The topological conjugacy relation $E_{t c}$ on the space $\mathcal{T}_{n}$ of Toeplitz flows is not smooth.

Of course, applying Proposition 2.1, this immediately implies the following result.

Corollary 4.3. The flip conjugacy relation $E_{f c}$ on the space $\mathcal{T}_{n}$ of Toeplitz flows is not smooth.

Clearly it is enough to prove Theorem 4.2 in the special case when $n=2$. For each $z \in 2^{\mathbb{N}}$, let $\tilde{z} \in 2^{\mathbb{Z}}$ be the corresponding Toeplitz sequence defined as follows.

- For each $m \geq 1$, let $B_{m}=\left[0,2^{m}-1\right]$ and suppose inductively that we have defined the value $\tilde{z}(\ell)$ for all integers $\ell \in B_{m} \backslash\left\{a_{m}, b_{m}\right\}$, where $0 \leq a_{m}<b_{m} \leq 2^{m}-1$. Let $c_{m}=a_{m}$ if $m$ is odd and $c_{m}=b_{m}$ if $m$ is even. Then we define

$$
\tilde{z}\left(c_{m}+k 2^{m}\right)=z(m-1) \quad \text { for all } k \in \mathbb{Z} .
$$

For example, at the beginning of stage 3 of the construction, $\tilde{z}\left\lceil B_{3}\right.$ is given by

$$
z(0) * z(0) z(1) z(0) * z(0) z(1)
$$

where the $*$ indicates that the value has not yet been defined. We then define $\tilde{z}(1+8 k)=z(2)$ for all $k \in \mathbb{Z}$ and hence obtain that for all $k \in \mathbb{Z}$,

$$
\tilde{z} \uparrow[8 k, 8(k+1))=z(0) z(2) z(0) z(1) z(0) * z(0) z(1)
$$


Notice that if $z \in 2^{\mathbb{N}}$ is an eventually constant sequence, then $\tilde{z}$ is a periodic sequence and so the corresponding orbit $\left\{\sigma^{m}(\tilde{z}) \mid m \in \mathbb{Z}\right\}$ is a finite closed subset of $2^{\mathbb{N}}$. From now on, let $\operatorname{Ec}\left(2^{\mathbb{N}}\right)$ be the set of eventually constant sequences $z \in 2^{\mathbb{N}}$ and let $\operatorname{Nec}\left(2^{\mathbb{N}}\right)=2^{\mathbb{N}} \backslash \operatorname{Ec}\left(2^{\mathbb{N}}\right)$.

Definition 4.4. Suppose that $x \in n^{\mathbb{Z}}$ is a Toeplitz sequence.

(i) For each $a \in \mathbb{Z}$, the corresponding minimal period $\operatorname{per}_{x}(a)$ is the least integer $b \geq 1$ such that $x(a+k b)=x(a)$ for all $k \in \mathbb{Z}$.

(ii) The set of essential periods of $x$ is defined to be $\left\{\operatorname{per}_{x}(a) \mid a \in \mathbb{Z}\right\}$.

Lemma 4.5. If $z \in \operatorname{Nec}\left(2^{\mathbb{N}}\right)$, then $\left\{2^{m} \mid m \in \mathbb{N}^{+}\right\}$is the set of essential periods of $\tilde{z}$.

Proof. With the above notation, it is enough to show that each $c_{m} \in B_{m}$ has minimal period $2^{m}$. This is clear when $m=1$. So suppose that $m>1$. Then at the beginning of stage $m$ of the construction, $\tilde{z}\left\lceil B_{m}\right.$ has the form $\bar{a} * \bar{b} \bar{a} * \bar{b}$, where $\bar{a} * \bar{b}$ has length $2^{m-1}$; and at the end of stage $m$, we know that $\tilde{z}$ has the form

$$
\cdots \bar{a} * \bar{b} \bar{a} z(m-1) \bar{b} \bar{a} * \bar{b} \bar{a} z(m-1) \bar{b} \bar{a} * \bar{b} \bar{a} z(m-1) \bar{b} \ldots
$$

Clearly $2^{m}$ is a period of $c_{m}$. Also, since $z$ is not eventually constant, we must eventually replace some $*$ by a value $z(\ell) \neq z(m-1)$ and so $2^{m-1}$ is not a period of $c_{m}$. Thus $c_{m}$ has minimal period $2^{m}$.

Definition 4.6. For each $z \in \operatorname{Nec}\left(2^{\mathbb{N}}\right)$, and $m \in \mathbb{N}^{+}$, let $W_{m}(\tilde{z})$ be the set of subsequences of $\tilde{z}$ of the form $\tilde{z} \uparrow\left[k 2^{m},(k+1) 2^{m}\right)$ for some $k \in \mathbb{Z}$.

Lemma 4.7. If $z \in \operatorname{Nec}\left(2^{\mathbb{N}}\right)$, then $\left|W_{m}(\tilde{z})\right|=2$ for all $m \in \mathbb{N}^{+}$.

Proof. If at the end of stage $m$ of the construction, $\tilde{z} \uparrow B_{m}$ has the form $\bar{c} * \bar{d}$, then $W_{m}(\tilde{z})=\{\bar{c} 0 \bar{d}, \bar{c} 1 \bar{d}\}$.

For each element $z \in \operatorname{Nec}\left(2^{\mathbb{N}}\right)$, let $X_{z} \in \mathcal{T}_{2}$ be the closure of the orbit $\left\{\sigma^{n}(\tilde{z}) \mid n \in \mathbb{Z}\right\}$ in $2^{\mathbb{Z}}$. Then it is clear that the map $z \mapsto X_{z}$ from $\operatorname{Nec}\left(2^{\mathbb{N}}\right)$ to $\mathcal{T}_{2}$ is Borel.

Proposition 4.8. If $y, z \in \operatorname{Nec}\left(2^{\mathbb{N}}\right)$ and $y E_{0} z$, then the Toeplitz flows $X_{y}$ and $X_{z}$ are topologically conjugate.

We will make use of the following result, which is a special case of Downarowicz-Kwiatkowski-Lacroix [7, Theorem 1]. 
Lemma 4.9. If $y, z \in \operatorname{Nec}\left(2^{\mathbb{N}}\right)$, then the following statements are equivalent:

(i) There exists a topological conjugacy $\pi: X_{y} \rightarrow X_{z}$ such that $\pi(\tilde{y})=\tilde{z}$.

(ii) For some $m \in \mathbb{N}^{+}$, there exists a bijection $\Pi: W_{m}(\tilde{y}) \rightarrow W_{m}(\tilde{z})$ such that

$$
\tilde{z}\left\lceil\left[k 2^{m},(k+1) 2^{m}\right)=\Pi\left(\tilde{y}\left\lceil\left[k 2^{m},(k+1) 2^{m}\right)\right)\right.\right.
$$

for all $k \in \mathbb{Z}$.

Proof of Proposition 4.8. Suppose that $y(m)=z(m)$ for all $m \geq m_{0}$; and suppose that at the end of stage $m_{0}$ of the constructions of $\tilde{y}, \tilde{z}$, we have that $\tilde{y}\left\lceil B_{m_{0}}=\bar{a} * \bar{b}\right.$ and $\tilde{z}\left\lceil B_{m_{0}}=\bar{c} * \bar{d}\right.$. Then $W_{m_{0}}(\tilde{y})=\{\bar{a} 0 \bar{b}, \bar{a} 1 \bar{b}\}$ and $W_{m_{0}}(\tilde{z})=\{\bar{c} 0 \bar{d}, \bar{c} 1 \bar{d}\}$. Furthermore, for any $k \in \mathbb{Z}$, the unique $*$ in the interval $\left[k 2^{m_{0}},(k+1) 2^{m_{0}}\right)$ is replaced at the same stage $m>m_{0}$ in the constructions of $\tilde{y}$ and $\tilde{z}$ with the value of $y(m-1)=z(m-1)$. Hence the map $\Pi: W_{m_{0}}(\tilde{y}) \rightarrow W_{m_{0}}(\tilde{z})$, defined by $\Pi(\bar{a} \varepsilon \bar{b})=\bar{c} \varepsilon \bar{d}$ for $\varepsilon=0,1$, satisfies statement (ii) of Lemma 4.9.

From now on, let $B=\left\{2^{m+1}-1 \mid m \in \mathbb{N}^{+}\right\}$and let $Z$ be the standard Borel subspace of $\operatorname{Nec}\left(2^{\mathbb{N}}\right)$ defined by

$$
Z=\left\{z \in \operatorname{Nec}\left(2^{\mathbb{N}}\right) \mid z(n)=0 \text { for all } n \in \mathbb{N} \backslash B\right\} .
$$

Clearly $E_{0} \uparrow Z$ is Borel bireducible with $E_{0}$.

Proposition 4.10. The Borel map $\theta: Z \rightarrow \mathcal{T}_{2}$ defined by $z \mapsto X_{z}$ is injective.

Combining Propositions 4.8 and 4.10, we see that the map $z \mapsto X_{z}$ is a weak Borel reduction from $E_{0} \uparrow Z$ to the topological conjugacy relation $E_{t c}$ on $\mathcal{T}_{2}$. Hence, applying Proposition 2.2, it follows that $E_{t c}$ is not smooth. This completes the proof of Theorem 4.2.

Proof of Proposition 4.10. Suppose that $y \neq z \in Z$. Then we can assume that $y(n)=0$ and $z(n)=1$, where $n$ is the least integer such that $y(n) \neq z(n)$. Let $n=2^{m+1}-1$ and let $s=2^{m}$. Suppose that at the end of stage $s$ of the constructions of $\tilde{y}, \tilde{z}$, we have that

$$
\tilde{y} \uparrow B_{s}=\bar{a} * \bar{b}=\tilde{z}\left\lceil B_{s} .\right.
$$

Then $W_{s}(\tilde{y})=W_{s}(\tilde{z})=\{\bar{a} 0 \bar{b}, \bar{a} 1 \bar{b}\}$ and $\tilde{y}, \tilde{z}$ are concatenations of the $2^{s}$-blocks $\bar{a} 0 \bar{b}$ and $\bar{a} 1 \bar{b}$. Let $t=2^{m+1}=2 s$ and consider $\tilde{z}\left\lceil B_{t}\right.$. Since 
$z(t-1)=z(n)=1$, it follows that the concatenation of $2^{s}$-blocks in $\tilde{z}$ contains a subsequence of period $2^{t} /\left|B_{s}\right|=2^{s}$ in which $\bar{a} 1 \bar{b}$ occurs. Similarly, since $z(s)=0$, the concatenation of $2^{s}$-blocks in $\tilde{z}$ contains a subsequence of period 2 in which $\bar{a} 0 \bar{b}$ occurs. Thus each occurrence of $\bar{a} 1 \bar{b}$ in the expression of $\tilde{z}$ as a concatenation of $2^{s}$-blocks is preceded and followed by occurrences of $\bar{a} 0 \bar{b}$. We claim that the sequence

$$
\underbrace{\bar{a} 0 \bar{b} \bar{a} 0 \bar{b} \cdots \bar{a} 0 \bar{b}}_{2^{s}+1 \text { times }}
$$

cannot occur as a subsequence of $\tilde{z}$. For suppose that the sequence $(4.10)$ occurs as the subsequence $\bar{u}=z_{k} \cdots z_{k+\left(2^{s}+1\right) 2^{s-1}}$ of $\tilde{z}$. Then $\bar{u}$ must contain $2^{s}$ consecutive $2^{s}$-blocks in the expression of $\tilde{z}$ as a concatenation of $2^{s}$-blocks, one of which must be $\bar{a} 1 \bar{b}$. However, the sequence (4.10) clearly repeats with period $2^{s}$ and so we must obtain two consecutive occurrences of the $2^{s}$-block $\bar{a} 1 \bar{b}$, which is impossible. On the other hand, since $y(\ell)=0$ for all $s \leq \ell \leq 4 s-2=2^{m+2}-2$, it follows easily that the sequence (4.10) occurs as a sub-block of $\tilde{y}$. Clearly this means that $X_{y} \neq X_{z}$.

\section{The proof of Theorem 1.4}

In this final section, we will present the proof of Theorem 1.4. Our argument involves the following variant of the Vitali equivalence relation $E_{0}$.

Definition 5.1. For each $x \in 2^{\mathbb{N}}$, let $\bar{x} \in 2^{\mathbb{N}}$ be the element defined by

$$
\bar{x}(n)=1-x(n) \quad \text { for all } n \in \mathbb{N} \text {. }
$$

Then $E_{0}^{*}$ is the countable Borel equivalence relation on $2^{\mathbb{N}}$ defined by

$$
x E_{0}^{*} y \quad \Longleftrightarrow \quad x E_{0} y \text { or } x E_{0} \bar{y} \text {. }
$$

Thus each $E_{0}^{*}$-class consists of exactly two $E_{0}$-classes. The proof of Theorem 1.4 makes use of the fact that there does not exist a Borel selection of an $E_{0}$-class within each $E_{0}^{*}$-class. ${ }^{\text {a }}$ For the sake of completeness, we have included a proof of this standard result.

Proposition 5.2. There does not exist a Borel homomorphism $\theta: 2^{\mathbb{N}} \rightarrow 2^{\mathbb{N}}$ from $E_{0}^{*}$ to $E_{0}$ such that $\theta(x) E_{0}^{*} x$ for all $x \in 2^{\mathbb{N}}$.

a first learned of this "standard measure-theoretic fact" from Coskey-Schneider [4]. 
Proof. Suppose that $\theta: 2^{\mathbb{N}} \rightarrow 2^{\mathbb{N}}$ is such a Borel homomorphism. Let $\mu$ be the usual product probability measure on $2^{\mathbb{N}}$ and let

$$
X=\left\{x \in 2^{\mathbb{N}} \mid x E_{0} y \text { for some } y \in \operatorname{ran} \theta\right\} .
$$

Then $X$ is a Borel tail event; and hence, by Kolmogorov's Zero-One Law, we have that $\mu(X)=0,1$. However, since the map $x \mapsto \bar{x}$ is measure preserving, it follows that $\mu\left(2^{\mathbb{N}} \backslash X\right)=\mu(X)$, which is impossible.

We are now ready to present the proof of Theorem 1.4. Suppose that there exists a Borel map $\varphi: \mathcal{G}_{f g} \rightarrow \mathcal{G}_{f g}$ such that for all infinite $G, H \in \mathcal{G}_{f g}$,

(i) $\varphi(G)$ is a just-infinite homomorphic image of $G$; and

(ii) if $G \cong H$, then $\varphi(G) \cong \varphi(H)$.

Applying Theorem 1.1 and Harrington-Kechris-Louveau [15], there exists a Borel reduction $z \mapsto S_{z}$ from $E_{0}$ to the isomorphism relation $\cong$ on the space of infinite finitely generated simple amenable groups. (The fact that $S_{z}$ is amenable will play no role in the proof of Theorem 1.4.) Consider the Borel map $\psi: 2^{\mathbb{N}} \rightarrow \mathcal{G}_{f g}$ defined by $z \mapsto G_{z}=S_{z} \times S_{\bar{z}}$. Since $S_{z}$ and $S_{\bar{z}}$ are nonabelian simple groups, the only nontrivial proper normal subgroups of $G_{z}$ are $S_{z}$ and $S_{\bar{z}}$; and it follows that:

(iii) $\psi$ is a Borel reduction from $E_{0}^{*}$ to $\cong$.

(iv) Each $\varphi\left(G_{z}\right)$ is isomorphic to either $S_{z}$ or $S_{\bar{z}}$.

Thus the Borel map $\theta: 2^{\mathbb{N}} \rightarrow 2^{\mathbb{N}}$ defined by

$$
\theta(z)=y \quad \Longleftrightarrow \quad y \in\{z, \bar{z}\} \text { and } \varphi\left(G_{z}\right) \cong S_{y}
$$

is a homomorphism from $E_{0}^{*}$ to $E_{0}$ such that $\theta(x) E_{0}^{*} x$ for all $x \in 2^{\mathbb{N}}$, which contradicts Proposition 5.2.

\section{Acknowledgments}

I would like to thank Alexander Kechris and the referee for some very helpful comments on earlier versions of this paper. The research in this paper was partially supported by NSF Grant DMS 1101597.

\section{References}

1. S. Bezuglyi and K. Medynets, Full groups, flip conjugacy, and orbit equivalence of Cantor minimal systems, Colloq. Math. 110 (2008), 409-429.

2. M. R. Bridson and A. Haefliger, Metric Spaces of Non-Positive Curvature, Grundlehren Math. Wiss. 319, Springer, Berlin, 1999. 
3. J. D. Clemens, Isomorphism of subshifts is a universal countable Borel equivalence relation, Israel J. Math. 170 (2009), 113-123.

4. S. Coskey and S. Schneider, Borel Cardinal Invariant properties of countable Borel equivalence relations, preprint (2011).

5. R. Dougherty, S. Jackson and A. S. Kechris, The structure of hyperfinite Borel equivalence relations, Trans. Amer. Math. Soc. 341 (1994), 193-225.

6. T. Downarowicz, Survey of odometers and Toeplitz flows, in: Algebraic and topological dynamics, Contemp. Math. 385, Amer. Math. Soc., Providence, 2005, pp. $7-37$.

7. T. Downarowicz, J. Kwiatkowski and Y. Lacroix, A criterion for Toeplitz flows to be topologically isomorphic and applications, Colloq. Math. 68 (1995), 219-228.

8. J. Feldman and C. C. Moore, Ergodic equivalence relations, cohomology and von Neumann algebras I, Trans. Amer. Math. Soc. 234 (1977), 289-324.

9. S. Gao, S. Jackson and B. Seward, A coloring property for countable groups, Math. Proc. Cambridge Philos. Soc. 147 (2009), 579-592.

10. S. Gao, S. Jackson and B. Seward, Group Colorings and Bernoulli Subflows, preprint (2011).

11. T. Giordano, I. F. Putnam and C. F. Skau, Full groups of Cantor minimal systems, Israel J. Math. 111 (1999), 285-320.

12. R. I. Grigorchuk, Degrees of growth of finitely generated groups and the theory of invariant means, Math. USSR-Izv. 25 (1985), 259-300.

13. R. I. Grigorchuk, Just infinite branch groups, in New Horizons in Pro-p Groups, Birkhäuser, Boston, 2000, pp. 121-179.

14. R. Grigorchuk and K. Medynets, Topological full groups are locally embeddable into finite groups, preprint 2012.

15. L. Harrington, A. S. Kechris and A. Louveau. A Glimm-Effros dichotomy for Borel equivalence relations. J. Amer. Math. Soc. 3 (1990), 903-927.

16. G. Hjorth and A. S. Kechris, Borel equivalence relations and classification of countable models, Annals of Pure and Applied Logic 82 (1996), 221-272.

17. S. Jackson, A.S. Kechris, and A. Louveau, Countable Borel equivalence relations, J. Math. Logic 2 (2002), 1-80.

18. K. Juschenko and N. Monod, Cantor systems, piecewise translations and simple amenable groups, preprint 2012.

19. A. S. Kechris Classical Descriptive Set Theory, Graduate Texts in Mathematics 156, Springer-Verlag, 1995.

20. H. Matui, Some remarks on topological full groups of Cantor minimal systems, Internat. J. Math. 17 (2006), 231-251.

21. A. Yu. Ol'shanski, SQ-universality of hyperbolic groups(Russian), Mat. Sb. 186 (1995), 119-132; translation in Sb. Math. 186 (1995), 1199-1211.

22. S. Thomas, Continuous versus Borel Reductions, Arch. Math. Logic 48 (2009), 761-770.

23. S. Thomas, Popa superrigidity and countable Borel equivalence relations, Annals Pure Appl. Logic. 158 (2009), 175-189.

24. S. Thomas and B. Velickovic, On the complexity of the isomorphism relation for finitely generated groups, J. Algebra 217 (1999), 352-373. 